\title{
La pertinence des politiques familiales municipales comme levier de développement social
}

\author{
Anne-Marie Tougas ${ }^{\mathrm{a}}, \mathrm{M}$. Ps., Université du Québec à Montréal \\ Lucie Fréchette, Ph. D., Université du Québec en Outaouais \\ Jacques Lizée ${ }^{b}$ - Carrefour action municipale et famille
}

\section{INTRODUCTION}

Le développement social est un concept qui prend de l'ampleur au Québec depuis les années 1970 avec le dépôt d'une première proposition de politique globale en la matière (Commission Castonguay-Nepveu, 1967-1972). En avril 1998, au terme d'une démarche préalable dans les régions, le Forum sur le développement social réunissait plus de 600 participants dans le but de faire progresser la compréhension collective du concept, de ce qu'il recouvre et des voies qui pourraient y conduire ${ }^{1}$. À ce jour, on fait référence à la définition proposée dans le cadre du Forum pour reconnaître et guider les initiatives porteuses de développement social :

« La mise en place et le renforcement, au sein des communautés, dans les régions et à l'échelle de la collectivité des conditions requises pour permettre, d'une part, à chaque individu de développer pleinement ses potentiels, de pouvoir participer activement à la vie sociale et de pouvoir tirer sa juste part de l'enrichissement collectif, et, d'autre part, à la collectivité de progresser socialement, culturellement et économiquement, dans un contexte où le développement économique s'oriente vers un développement durable, soucieux de justice sociale. »

Le concept de développement social est complexe, notamment parce qu'il renvoie à plusieurs idées et valeurs sujettes à des interprétations variables ${ }^{2}$ et qu'il rapporte autant à une finalité qu'à un processus ${ }^{3}$. Par ailleurs, ce concept apparaît à la fois distinct et complémentaire à d'autres types d'initiatives promues dans différents programmes et politiques publiques : développement des communautés, développement local, développement durable, développement des territoires, etc.
D'abord envisagé à l'échelle nationale, le développement social est associé à l'élaboration de politiques traitant de questions sociales sous divers thèmes (santé, pauvreté, culture, logement, loisir, etc.). Sous l'angle territorial, il fait aussi partie du paysage régional et municipal. Depuis longtemps le code municipal indiquait des compétences possibles dans des champs d'intervention associés au développement social. C'est toutefois avec la décennie 2000-2010 que le développement social a gagné du terrain de façon remarquable à l'échelle municipale. Le législateur en a fait une compétence obligatoire dans les nouvelles grandes villes du Québec (Loi 170) puis dans les MRC avec la révision du plan d'aménagement. Les municipalités doivent donc désormais planifier le développement de leur territoire en tenant compte tant de ses facettes sociales, qu'économiques ou communautaires.

\section{À l'échelle nationale, le développement social est associé à l'élaboration de politiques traitant de questions sociales sous divers thèmes (santé, pauvreté, culture, logement, loisir, etc.).}

Cette nouvelle responsabilité d'intégrer la dimension sociale dans les actions en développement du territoire peut représenter un défi de taille pour les élus. Les politiques en la matière sont récentes, il n'existe pas de consensus clair quant à la définition du concept et les modalités de reddition de compte ne sont pas précisées. En conséquence, la confusion dans la compréhension de la Loi, la crainte face au transfert de responsabilités par le gouvernement et l'association fréquente du développement social aux services aux populations démunies risquent de 
freiner les municipalités dans leurs actions en la matière ${ }^{4}$. Il n'en demeure pas moins que les municipalités possèdent déjà de nombreux acquis sur le plan des politiques traitant de questions sociales, notamment dans des champs tels la culture, le loisir, l'habitation et la famille. Ce dernier champ, précisément celui des politiques familiales municipales, nous intéresse plus spécifiquement.

\section{LES POLITIQUES FAMILIALES MUNICIPALES ET LE DÉVELOPPEMENT SOCIAL}

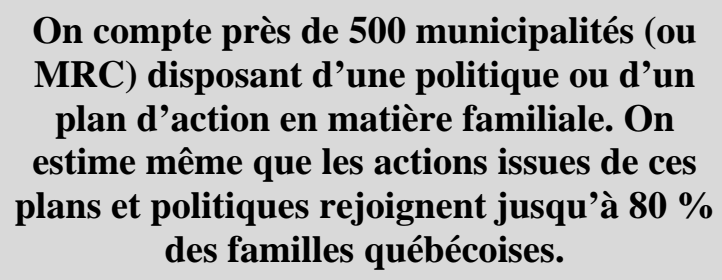

On compte près de 500 municipalités (ou MRC) disposant d'une politique ou d'un plan d'action en matière familiale. On estime même que les actions issues de ces plans et politiques rejoignent jusqu'à $80 \%$ des familles québécoises.

Depuis 1988, les politiques familiales s'implantent graduellement dans l'univers municipal québécois. En appui à leurs démarches en ce sens, les municipalités (ou MRC) peuvent compter sur le Carrefour action municipale et famille (CAMF), un organisme communautaire dont la mission consiste à offrir des services professionnels et techniques, de promotion, de soutien et d'accompagnement des politiques familiales municipales (PFM). Dès l'émergence d'un projet de politique familiale municipale, le CAMF propose un rapprochement entre citoyens et élus, s'actualisant par la formation d'un comité de pilotage. Ce comité agit à titre consultatif en matière de besoins des familles du territoire et au regard des actions à conduire pour les satisfaire, selon les différents champs d'intervention de la municipalité : habitation, loisirs, santé, transport, service de garde, environnement, sécurité, travail, etc. Depuis ses débuts, la démarche proposée trouve un nombre croissant de preneurs. Si bien qu'à ce jour, on compte près de 500 municipalités (ou MRC) disposant d'une politique ou d'un plan d'action en matière familiale. On estime même que les actions issues de ces plans et politiques rejoignent jusqu'à $80 \%$ des familles québécoises ${ }^{5}$.

L'originalité des politiques familiales municipales comme initiative participante du développement social ne fait aucun doute. Le sujet a été documenté en décrivant ces politiques sous l'angle de leurs objectifs, de leurs principes directeurs ou de leurs orientations ${ }^{6}$. Dans la démarche suggérée pour l'élaboration de la politique familiale municipale ${ }^{7}$, plusieurs éléments indiquent déjà une interface certaine avec le développement social. Plus spécifiquement, les objectifs généraux de la démarche évoquent notamment la nécessité d'instaurer et de développer la culture du «penser et agir famille », d'accroître le sentiment d'appartenance à la communauté, de stimuler l'interaction avec la collectivité et d'améliorer la qualité des milieux de vie des familles. Du côté des retombées, on projette, entre autres choses, d'améliorer l'accessibilité aux services de proximité, le sentiment de sécurité, le soutien aux familles défavorisées et la stimulation de l'économie locale; des thèmes qui rejoignent aussi les préoccupations associées au développement social.

Pour compléter le portrait des interfaces entre politique familiale municipale et développement social, il restait à vérifier comment les municipalités traduisent leurs politiques familiales en actions dans une optique de développement social. Ainsi, nous posions l'hypothèse que la politique familiale municipale, par les mesures qui émergent des plans d'action, contribuent non seulement au développement social des municipalités mais ouvre également une avenue favorable à la participation des citoyens dans leur communauté. C'est précisément l'angle d'étude de cet article qui prend appui sur une recherche initiée par le CAMF dans le cadre des activités de l'ARUC-ISDC.

Suivant une démarche inductive s'appuyant sur la littérature dans le domaine, nous avons d'abord procédé à la définition et à la validation de cinq stratégies décrivant l'action municipale en matière de développement social. Ces stratégies s'inscrivent dans une perspective qui reconnaît la participation des citoyens en tant que processus et finalité attendus du développement social. Les stratégies sont ordonnées de façon à situer les divers modes de contribution 
au développement social en prenant en compte le degré de participation sollicité auprès des citoyens dans l'amélioration de la qualité de vie de leur milieu. Le schéma 1 illustre le modèle sur lequel s'appuie l'analyse des mesures issues des plans d'action de la politique familiale municipale.

Les deux premières stratégies, offre de ressources et accès aux ressources, contribuent au développement social avec des activités pour lesquelles on attribue généralement un rôle passif aux citoyens. Les modalités qui favorisent l'accès aux ressources municipales dans une perspective de développement social rejoignent les grands vecteurs de l'accessibilité, tels qu'élaborés en contexte de loisirs ${ }^{8,9}$ : temporel, spatial, économique, culturel et social. La troisième, nformation/promotion/sensibilisation, est une stratégie qui demande aux citoyens d'être davantage proactifs dans la consommation de l'information mise à leur disposition par la municipalité ou ses partenaires sociaux. Elle outille le citoyen pour mieux profiter des services municipaux, pour recueillir des données enrichissant son bien-être personnel ou pour participer plus activement à la vie de sa communauté locale. On en arrive ensuite à des stratégies de développement social qui se rapprochent de la participation active et de la mobilisation dans le milieu. D'une part, par le soutien aux initiatives du milieu, les municipalités stimulent l'initiative collective de renforcement des communautés locales. De cette façon, les municipalités reconnaissent le rôle essentiel des organisations dans la mise en réseau des citoyens et contribuent à offrir un terreau favorable à l'exercice du pouvoir dans une optique de solidarité collective et de participation citoyenne. Enfin, la stratégie consultation/représentation confère un rôle ou un mode de représentation actif aux citoyens dans la prise de décisions face aux différentes problématiques de leur municipalité. Cette dernière stratégie permet également une prise en compte des besoins de la population, essentielle à la réflexion entourant la mise en œuvre des quatre stratégies précédentes. On peut donc parler d'une brochette de stratégies réparties sur un continuum allant du rôle passif du citoyen jusqu'à son rôle actif dans l'actualisation d'actions associées au développement social local.

\section{Schéma 1 : Continuum des stratégies de développement social municipal}

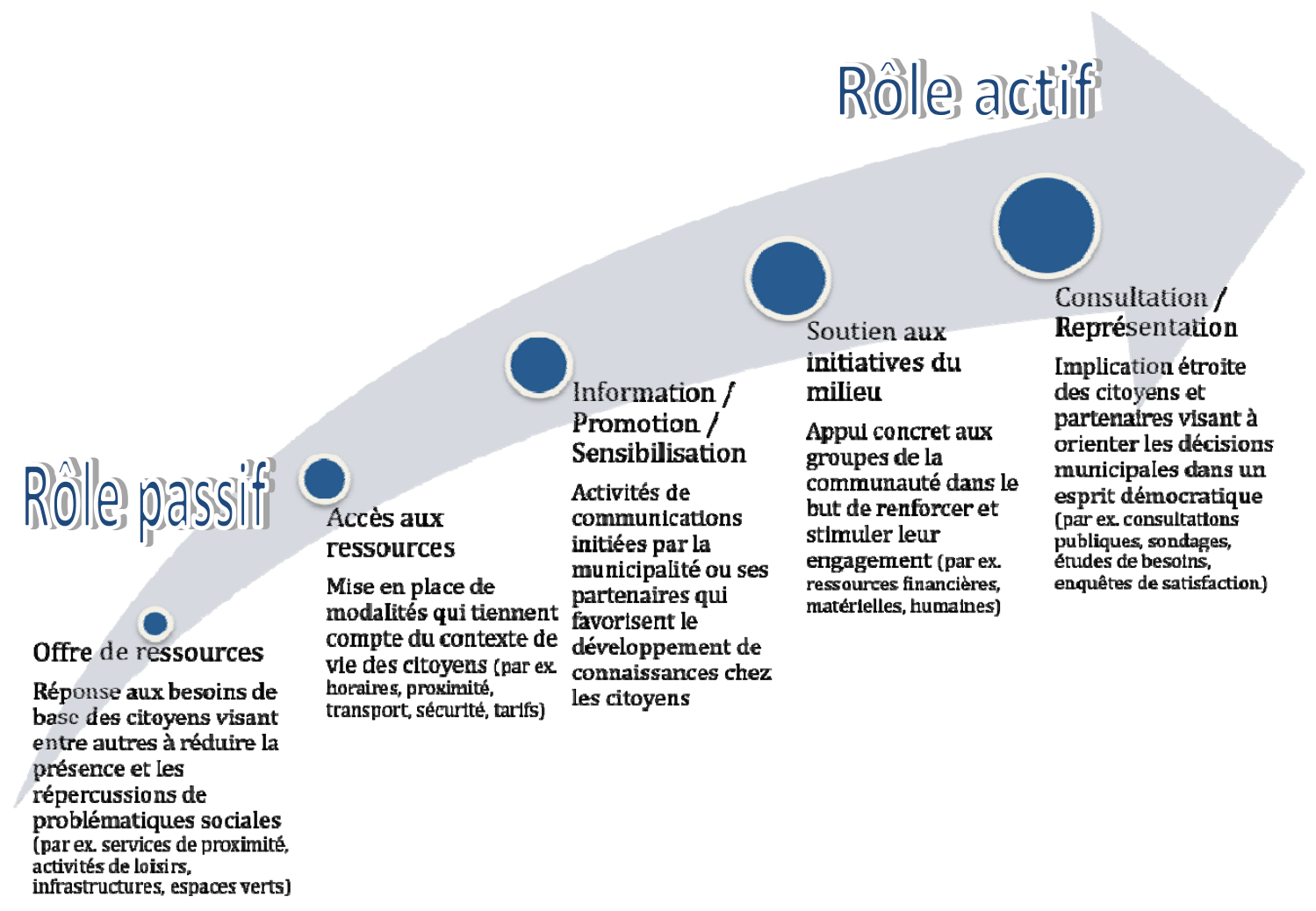




\section{REGARD SUR LES MESURES ISSUES DES PLANS D'ACTION DE PFM}

\subsection{Des données en cohérence avec le développement social}

L'analyse de 88 plans d'actions de politiques familiales municipales élaborés entre 2006 et 2008 a fourni 2853 mesures dont $96 \%$ peuvent être associées à l'une ou l'autre des stratégies précédemment décrites. On retrouve plus des trois quarts des actions proposées sous trois des cinq stratégies identifiées comme propices au développement social : l'accès aux ressources $(27 \%)$, l'information/promotion/sensibilisation $(26 \%)$ et l'offre de ressources $(25 \%)$. Les stratégies de soutien aux initiatives du milieu $(15 \%)$ et de consultation/représentation $(7 \%)$ comptent parmi les moins populaires au sein des politiques familiales municipales. Le tableau 1 présente la répartition de cette couverture et l'illustre à l'aide d'exemples tirés des mesures de politiques familiales municipales analysées.

\section{Tableau 1 - Couverture des stratégies de DS dans les plans d'action de PFM}

\begin{tabular}{|c|c|}
\hline $\begin{array}{c}\text { Accès aux } \\
\text { ressources }(27 \%)\end{array}$ & $\begin{array}{l}\text { - Modifier la réglementation pour favoriser la création d'habitations intergénérationnelles } \\
\text { - Faciliter l'intégration d'enfants handicappés aux activités municipales } \\
\text { - Offrir le transport gratuit pour les enfants fréquentant les terrains de jeux } \\
\text { - Prévoir des aménagements réservés aux jeunes familles }\end{array}$ \\
\hline $\begin{array}{l}\text { Information } \\
\text { Promotion } \\
\text { Sensibilisation } \\
\quad(26 \%)\end{array}$ & $\begin{array}{l}\text { - Faire connaître les mesures de soutien aux familles hébergeant des personnes proches } \\
\text { - Informer les courtiers et agents d'immeuble des orientations de la municipalité } \\
\text { - Sensibiliser les jeunes sur l'engagement social } \\
\text { - Écrire un article mensuel sur l'importance du recyclage et de la récupération }\end{array}$ \\
\hline $\begin{array}{l}\text { Offre de ressources } \\
\qquad(25 \%)\end{array}$ & $\begin{array}{l}\text { - Poursuivre l'aménagement d'espaces communautaires dans les HLM } \\
\text { - Offrir un accès Internet aux familles à la bibliothèque } \\
\text { - Offrir le service de garde avant et après l'école } \\
\text { - Poursuivre l'aménagement du réseau cyclable }\end{array}$ \\
\hline $\begin{array}{l}\text { Soutien aux } \\
\text { initiatives du } \\
\text { milieu }(15 \%)\end{array}$ & $\begin{array}{l}\text { - Faciliter la mise en place de coopératives d'habitation } \\
\text { - Assurer le prêt de locaux aux organismes lors d'activités familiales } \\
\text { - Impliquer directement les jeunes dans l'organisation de leurs activités } \\
\text { - Développer et soutenir la médiation de quartier }\end{array}$ \\
\hline $\begin{array}{l}\text { Consultation } \\
\text { Représentation } \\
(7 \%)\end{array}$ & $\begin{array}{l}\text { - Organiser des rencontres de secteur sur les besoins des familles } \\
\text { - Poursuivre la collecte de suggestions dans les boîtes installées à cet effet } \\
\text { - Créer un comité des organismes } \\
\text { - Organiser une rencontre annuelle des responsables de questions familiales }\end{array}$ \\
\hline
\end{tabular}

De prime abord, on constate que les plans d'actions des politiques familiales municipales abordent une diversité de stratégies propices au développement social. Si on s'intéresse d'un peu plus près au degré auquel la participation des citoyens est encouragée par les mesures des politiques familiales municipales analysées, on constate que la moitié des actions qui en sont issues $(52 \%)$ la sollicitent à un degré faible, c'està-dire qu'elles se rattachent aux stratégies offre de ressources et accès aux ressources. À un degré moyen, vient ensuite la stratégie information/promotion/sensibilisation (26\%), laquelle recèle le potentiel d'outiller les citoyens non seulement en fonction de leur bien-être mais encore en fonction de leur participation éclairée à la vie sociale et politique municipale. Finalement, on constate que les stratégies qui encouragent davantage la participation des citoyens, soutien aux initiatives du milieu et consultation/représentation, ne comptent que pour $22 \%$ de l'ensemble des actions issues des politiques familiales municipales. D'ailleurs, la stratégie la moins présente dans les plans d'action est justement celle qui sollicite le plus la participation des citoyens (consultation/représentation : $7 \%$ ).

\section{Les plans d'actions des politiques familiales municipales abordent une diversité de stratégies propices au développement social.}




\subsection{Les résultats relus à la lumière de la taille des municipalités}

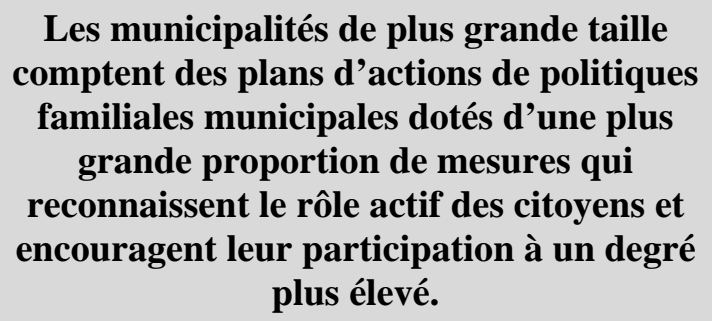

Les municipalités de plus grande taille comptent des plans d'actions de politiques familiales municipales dotés d'une plus grande proportion de mesures qui reconnaissent le rôle actif des citoyens et encouragent leur participation à un degré plus élevé.

Une lecture des résultats de la recherche sous l'angle quantitatif indique que les municipalités de plus grande taille comptent des plans d'actions de politiques familiales municipales dotés d'une plus grande proportion de mesures qui reconnaissent le rôle actif des citoyens et encouragent leur participation à un degré plus élevé. Plus précisément, les municipalités de 50000 habitants et plus conduisent davantage d'actions se rapportant au soutien du milieu et à la consultation/représentation, comparativement aux municipalités de plus petites tailles. Quand on scrute la nature des mesures plutôt que leur nombre, on voit que les municipalités de plus petites tailles mettent davantage l'accent sur des actions qui sollicitent faiblement la participation des citoyens. Ainsi l'offre de ressources est la stratégie la plus fréquente dans les municipalités de 1 à 4999 habitants et cela est aussi vrai dans celles comptant de 10000 à 19999 habitants.

On peut faire l'hypothèse que plusieurs milieux de plus petite taille souhaiteraient élargir la palette des services disponibles pour les citoyens dans leur territoire même, plutôt que de voir ceux-ci devoir recourir à des ressources ailleurs. La rétention de la population, spécialement celle des jeunes familles, en est souvent tributaire et les élus sont sensibles aux souhaits des citoyens en ce sens. La présence de services de proximité fait partie des éléments définissant la qualité de vie et qui influent sur la perception que les gens ont de l'intérêt à développer leur communauté locale et à $\mathrm{y}$ vivre.

Du côté des grandes villes, au moins dans le cas des nouvelles grandes agglomérations, un découpage par arrondissements, par villages urbains ou autres formes de découpage administratif induit une dynamique sociopolitique municipale diffé- rente. Ce découpage ne saurait être fonctionnel sans qu'on y trouve un minimum de dispositifs de consultation de la population. D'ailleurs, plusieurs grandes villes (dont Montréal, Québec, Gatineau et Sherbrooke) ont déjà adopté une politique en $\mathrm{Ce}$ sens. Est-ce à dire que le développement social, dans la mesure où on encourage la participation comme élément de processus et de finalité, est mieux desservi dans les municipalités de grande taille? On ne peut pas tirer de conclusion en ce sens mais indiquer que les dispositifs officiels de consultation recèlent le potentiel de maximiser la participation de la population. L'usage qu'on en fait demeure cependant fort variable d'une municipalité à l'autre et mériterait d'être étudié plus en profondeur pour déterminer les pratiques plus profitables pour les citoyens comme pour les municipalités.

Les petites municipalités profitent quant à elles d'une dynamique différente pour encourager la participation des citoyens étant donnée la proximité engendrée par la taille de leur population. Le même phénomène peut se reproduire à l'échelle des quartiers peu populeux dans des villes de taille moyenne. La plus faible présence de dispositifs formalisés encourageant le rôle actif des citoyens, tel qu'observé dans la présente étude, ne signifie toutefois pas que la participation ou consultation n'a pas cours ni que le développement social ne soit pas pris en compte par les municipalités. Une recherche ${ }^{10}$ sur les dépenses en développement social démontre bien que l'investissement en développement social s'est nettement accru entre 2000 et 2006 et ce, pour les municipalités de toutes tailles. Certaines municipalités de moyenne ou petite taille ont même accru leur niveau d'effort de façon plus importante que l'ont fait les grandes villes.

L'investissement en développement social s'est nettement accru entre 2000 et 2006 et ce, pour les municipalités de toutes tailles. Certaines municipalités de moyenne ou petite taille ont même accru leur niveau d'effort de façon plus importante que l'ont fait les grandes villes. 
Ces données reflètent une préoccupation bien ancrée chez les élus ou les responsables des questions familiales dans les municipalités quant à l'offre de services assurant le bien-être des citoyens et une qualité de vie favorable au développement des familles de leur territoire. Les stratégies adoptées à cet effet semblent cependant relever plus souvent d'une initiative de l'appareil municipal plutôt que de stratégies sollicitant le rôle actif des citoyens. On note toutefois que le soutien aux initiatives du milieu tempère cette observation en laissant place à des stratégies où la concertation et le partenariat peuvent être au cœur du développement et de l'accès aux services. Il paraît toutefois que les plans d'action ne valorisent pas la mobilisation des citoyens ou leur participation active aux processus de décisions dans une perspective favorable au développement social. Il s'agit là d'une donnée qui mérite des analyses plus approfondies pour la transformer en défi à relever. C'est l'optique avec laquelle nous scrutons plus avant le potentiel de levier des politiques familiales municipales pour aller au-delà des constats issus de cette recherche.

\section{COMMENT ACCROÎTRE L'EFFET DE LEVIER DES POLITIQUES FAMILIALES MUNICIPALES SUR LE DÉVELOPPEMENT SOCIAL EN CONTEXTE MUNICIPAL?}

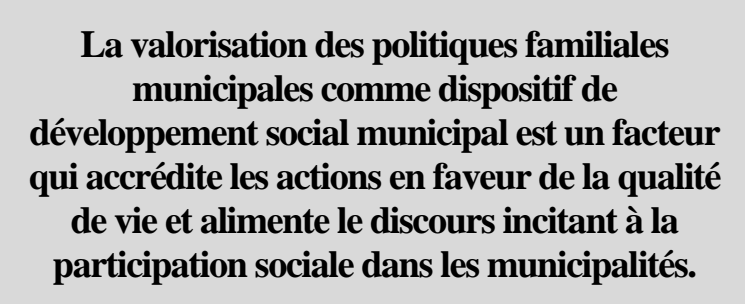

Les politiques familiales municipales représentent un levier intéressant pour promouvoir le développement social d'une communauté. Comment les municipalités peuvent-elles accrồtre cet effet de levier? Nous ne détenons pas de données permettant de vérifier dans quelle proportion les mesures annoncées dans les plans d'actions issus des politiques familiales municipales ont été réalisés ni à quel degré elles l'ont été. Nous pouvons cependant proposer des facteurs facilitant issus des échanges des chercheurs avec les élus et les comités soutenant la démarche d'élaboration des politiques familiales municipales et à partir de l'expérience du CAMF qui est engagé dans le développement des politiques familiales municipales depuis plus de 20 ans. Pour les fins de cet article, trois facteurs sont retenus: la valorisation des politiques familiales municipales comme structures intégratrices de développement social, la mise en œuvre de dispositifs de suivi soucieux de la transversalité entre les politiques et l'attribution de ressources nécessaires.

La valorisation des politiques familiales municipales comme dispositif de développement social municipal est un facteur qui accrédite les actions en faveur de la qualité de vie et alimente le discours incitant à la participation sociale dans les municipalités. Ceci implique toutefois que le conseil municipal accorde de l'importance à ce dossier en le confiant à un élu, nommé par le fait même responsable des questions familiales (RQF), qui fait rapport régulièrement au conseil et entretient des liens avec les organisations concernées par l'action en faveur des familles. En faire une responsabilité officielle donne du poids à ce dossier et lui confère une place dans les débats au sein des instances politiques et administratives municipales.

Pour favoriser la mise en œuvre de tout plan d'action, des dispositifs de suivi s'avèrent précieux. Dans le cas des politiques familiales municipales, on peut penser à faire appel à des comités de suivi un peu à la manière des comités de pilotage dont l'action a préalablement jalonné la démarche d'élaboration des politiques familiales municipales. On peut également penser à des activités annuelles ou triennales, coordonnées par ces comités de suivi, pour évaluer l'avancement de l'action en faveur des familles et du développement de la qualité de vie dans la municipalité. Dans le même esprit, de façon à ce que les actions en faveur du développement social s'additionnent dans une optique transversale, on peut penser à la création d'un comité d'harmonisation des mesures au sein de l'appareil municipal. Parmi les politiques traitant de la dimension sociale du développement municipal, la politique familiale municipale demeure probablement la plus répandue à 
ce jour. Selon les milieux s'ajoutent des politiques ou planifications en loisir, en vie communautaire, en culture ou en développement social en soi. L'interface entre ces politiques révèle qu'une partie de leurs intentions eu égard au développement de la qualité de vie du milieu se rejoignent. Le défi est de faire en sorte que les intervenants actifs dans chaque dossier coordonnent leurs efforts pour que ceux-ci s'additionnent plutôt que de les voir entrer en compétition les uns avec les autres.

Finalement, on ne saurait éviter la question de l'investissement. De toute évidence, les actions mises en place par les municipalités sont tributaires des ressources matérielles, humaines et financières dont elles disposent. Celles-ci leur donnent les moyens de leurs ambitions. Les municipalités dotées de politiques familiales municipales expriment d'ailleurs la crainte de ne

\section{CONCLUSION}

\section{Au cours des prochaines années, de nouvelles politiques familiales municipales de deuxième et troisième générations verront le jour, ce qui laisse espérer un espace démocratique marqué par l'accrois- sement de la participation citoyenne.}

Bien que les politiques familiales municipales fassent partie du paysage municipal québécois depuis plus de deux décennies, l'expérience acquise en la matière demeure relativement récente. Cela pourrait expliquer la tendance des municipalités à répondre d'abord et avant tout aux besoins des familles en offrant de nouvelles ressources, en les rendant accessibles ou en en faisant la promotion. L'ouverture à la participation comme élément indissociable du développement social passe maintenant davantage dans le discours et commence à faire son chemin au sein des plans d'action issus des politiques familiales municipales. On note une tendance dans les municipalités à mettre graduellement en place des mécanismes favorables à un apprivoisement mutuel entre élus et organisations porteuses d'une tradition en ce sens sur leur territoire. Il est certes possible de relever le défi de maximiser la pas pouvoir réaliser autant qu'elles le souhaiteraient les mesures élaborées dans leurs plans d'actions ${ }^{11}$. Une partie de l'effort financier en développement social relève de la municipalité qui a elle-même des choix à effectuer dans la préparation du budget. On ne saurait par contre oublier que pour actualiser la compétence conférée par le législateur aux grandes municipalités et MRC, celles-ci auraient besoin de moyens financiers accrus de la part des autres paliers de gouvernement, ce qu'elles réclament de façon récurrente. La participation a elle aussi un coût si l'on souhaite qu'elle s'effectue dans des conditions qui la rendent réelle et efficiente. Il s'agit souvent là d'une dimension occultée lors de la préparation de politiques ou de plans d'action. L'intention et le discours sont à la participation, mais l'on oublie trop souvent l'investissement qu'elle requiert.

participation des citoyens si on y met le temps et les moyens. Au cours des prochaines années, de nouvelles politiques familiales municipales de deuxième et troisième générations verront le jour, ce qui laisse espérer un espace démocratique marqué par l'accroissement de la participation citoyenne.

\section{BIBLIOGRAPHIE}

${ }^{1}$ Conseil de la santé et du bien-être (1997). Forum sur le développement social : Présentation de la démarche. Québec: Gouvernement du Québec.

${ }^{2}$ Robert, L. ( 2006). Développement social. Une politique sociale en émergence? Dans M. Tremblay, P.-A. Tremblay et S. Tremblay, Le développement social. Un enjeu pour l'économie sociale. Sainte-Foy, PUQ : 49-65.

${ }^{3}$ Réseau québécois de développement social (2009). La question de la définition du développement social. Site internet accédé le 17 mars 2009,

${ }^{4}$ Letarte, G., Fréchette, L., Bourque, D. Lizée, J. (2007). Les interfaces entre les politiques municipales : développement social, famille, loisir, sport et vie communautaire. Gatineau, Cahiers de l'ARUC-ISDC, UQO, $88 \mathrm{p}$.

${ }^{5}$ Lizée, J. (2008). Les chiffres nous parlent. Bulletin municipalité et famille, 6(1): 9 .

${ }^{6}$ Fréchette, L. (2006). Le développement social à l'échelle municipale. Dans M. Tremblay, P.-A. Tremblay et S. Tremblay, Le développement social. Un enjeu pour l'économie sociale. Sainte-Foy, PUQ: 132-146. 
${ }^{7}$ Ministère de la Famille et des Aînés (2007). Regard sur la démarche relative à l'élaboration ou à la mise à jour d'une politique familiale municipale. Le programme de soutien financier et technique aux municipalités et aux municipalités régionales de comté. Québec: Gouvernement du Québec.

${ }^{8}$ Jolin, L. (2005). Projet de grille d'analyse de l'accessibilité au loisir. Communication présentée au Forum québécois du loisir 2005, ARUC en économie sociale, UQÀM, Montréal, 35 p. ${ }^{9}$ Fréchette, L. ( 2007). L'accessibilité au loisir par la lorgnette des centres communautaires de loisir. Cahier Céris, UQO, 15p. ${ }^{10}$ Letarte, G.\& Fréchette, L. (2009). L'investissement en développement social dans les municipalités québécoises après les fusions municipales. Étude de l'évolution des dépenses municipales dans les champs du loisir, de la culture et $d u$ logement social. Gatineau, Cahier de l'ARUC-ISDC, UQO, 74p. ${ }^{11}$ Ministère de la Famille et des Aînés (2008). Rapport synthèse. Évaluation du programme de soutien financier et technique aux municipalités et aux MRC pour l'élaboration ou la mise à jour d'une politique familiale municipale. Québec: Gouvernement du Québec.

\section{NOTES}

aDoctorante en psychologie communautaire, Université du Québec à Montréal.

birecteur de la recherche et de la formation, Carrefour action municipale et famille et Co-responsable de l'axe développement social des communautés, ARUC-ISDC. 